\title{
Ideal spin hydrodynamics from Wigner function approach
}

\author{
Hao-Hao Peng, ${ }^{1,2}$ Jun-Jie Zhang, ${ }^{3}$ Xin-Li Sheng, ${ }^{4}$ and Qun Wang ${ }^{1,2}$ \\ ${ }^{1}$ Interdisciplinary Center for Theoretical Study and Department of Modern Physics, \\ University of Science and Technology of China, Hefei, Anhui 230026, China \\ ${ }^{2}$ Peng Huanwu Center for Fundamental Theory, Hefei, Anhui 230026, China \\ ${ }^{3}$ Northwest Institute of Nuclear Technology, Xi'an 710024, China \\ ${ }^{4}$ Key Laboratory of Quark and Lepton Physics (MOE) and Institute of Particle Physics, \\ Central China Normal University, Wuhan, 430079, China
}

\begin{abstract}
Based on the Wigner function in local equilibrium, we derive hydrodynamical quantities for a system of polarized spin-1/2 particles: the particle number current density, the energy-momentum tensor, the spin tensor, and the dipole moment tensor. Comparing with ideal hydrodynamics without spin, additional terms at first and second order in the Knudsen number Kn and the average spin polarization $\chi_{s}$ have been derived. The Wigner function can be expressed in terms of matrixvalued distributions, whose equilibrium forms are characterized by thermodynamical parameters in quantum statistics. The equations of motions for these parameters are derived by conservation laws at the leading and next-to-leading order $\mathrm{Kn}$ and $\chi_{s}$.
\end{abstract}

Introduction. Large orbital angular momenta (OAM) perpendicular to the reaction plane can be generated in noncentral relativistic heavy ion collisions. Part of initial OAM are converted to spin angular momenta of quarks through the spin-orbit coupling $[1,2]$, similar to the Einstein-de Hass and Barnett effects [3, 4]. At the freeze-out stage, these polarized quarks combine to form polarized hadrons. The polarization of hyperons along the global OAM (global spin polarization) has recently been measured in the STAR experiment $[5,6]$. According to quantum statistics [7-9], the spin polarization is connected to the thermal vorticity $\omega_{\mu \nu}^{\text {th }} \equiv-\left(\partial_{\mu} \beta_{\nu}-\partial_{\nu} \beta_{\mu}\right) / 2$, where $\beta_{\mu}=u_{\mu} / T$ is the ratio of the flow velocity $u_{\mu}$ to the temperature $T$. Hydrodynamical and transport models [10-14] can describe the experimental data of the global $\Lambda$ polarization. However, the longitudinal polarization of $\Lambda \mathrm{s}$ calculated in hydrodynamical and transport models [15] has an opposite sign to experimental results [16], which is called a "sign problem". Several attempts have been made in explaining the "sign problem", including different choices of spin potentials [17], non-relativistic connection between the polarization and vorticity [18], off-equilibrium contributions according to transport model simulations [19], the contribution from the shear-tensor [20-23], and the equation of states and freeze-out temperatures [24], but at present no definite conclusion has been drawn. Meanwhile, it is expected to exist a parity violation in a hot QCD matter, which may be observed through the Chiral Magnetic Effect (CME) [25-27]. Isobar collisions have been carried out by the STAR collaboration [28-30] but no CME signatures have been observed [31]. In order to better understand the "sign problem" and search for the CME signature, more theoretical efforts and numerical simulations for the dynamical evolution of spin degrees of freedom are needed, see e.g. Refs. [32-35] for recent reviews of spin effects in heavy ion collisions.

One way to describe the evolution of spin degrees of freedom in the quark gluon plasma is through relativistic spin hydrodynamics. Relativistic viscous hydrodynamics (without spin) is a successful framework to describe the evolution of strong interaction matter in heavy-ion collisions and therefore is widely used in numerical simulations [36-41]. Comparing with the spinless case, the spin hydrodynamics includes an additional conservation law for the total angular momentum, which allows a conversion between spin and OAM. The spin hydrodynamics was proposed many years ago [42]. Recent developments in the relativistic hydrodynamics include the effective theory approach [43-45], the derivation using the local equilibrium distribution functions [46-49] introduced in [9]. The second order hydrodynamical equations can be derived in many approaches, such as the generating function method [50], relaxation time approximation [51, 52], and entropy current analysis based on the second law of thermodynamics [53-56].

In this work, we will derive relativistic spin hydrodynamics for ideal fluids from Wigner functions. The conventional ideal hydrodynamics can be recovered in the spinless case. The Wigner function is expressed in terms of matrix-valued distributions, whose local equilibrium forms are given by quantum statistics [9]. We first obtain the Wigner function up to the second order in space-time derivatives by solving the equation of the Wigner function. From the Wigner function, we derive hydrodynamical quantities as functions of thermodynamical parameters: the temperature, the flow velocity, the chemical potential and the spin potential. The equations of motions for these parameters are obtained from conservation laws.

Throughout this work, we use natural units $\hbar=c=$ $k_{B}=1$. The antisymmetric tensor is defined as $A^{[\mu} B^{\nu]} \equiv$ $\left(A^{\mu} B^{\nu}-A^{\nu} B^{\mu}\right) / 2$, while the symmetric one is defined as $A^{\{\mu} B^{\nu\}} \equiv\left(A^{\mu} B^{\nu}+A^{\nu} B^{\mu}\right) / 2$. 
Power counting. Before we start our discussion, we want to clarify the power counting scheme used throughout this letter. In an ordinary Navier-Stokes type of fluid, it is usually assumed that the hydrodynamical limit can be reached when microscopic details in the system can be safely neglected. This limit is quantified as $\mathrm{Kn} \equiv l / L \ll 1$, where the Knudsen number $\mathrm{Kn}$ is defined as the ratio of the typical microscopic length scale $l$ to the macroscopic length scale $L$. In our discussion, $l$ is associated with the microscopic mean-free-path, while $L$ is described by $1 / L \sim\left|\partial_{\mu} O / O\right|$, the typical scale of the gradient of the macroscopic quantity $O$, such as the charge and energy densities, etc. Therefore a gradient expansion is equivalent to an expansion with respect to the Knudsen number.

For systems with spin degrees of freedom, we introduce an additional parameter $\chi$ to quantify spin polarization. This parameter can be given by either the average spin polarization per particle or the average magnetic dipole moment,

$$
\chi_{s} \sim \frac{\left|\mathcal{S}^{\lambda, \mu \nu}\right|}{n} \sim \frac{\left|\mathcal{M}^{\mu}\right|}{n},
$$

where $\mathcal{S}^{\lambda, \mu \nu}$ is the spin tensor density, $\mathcal{M}^{\mu}$ is the magnetic dipole moment density, and $n$ is the particle number density. If spin effects are induced by the thermal vorticity as in global equilibrium, we expect $\chi_{s} \sim \mathrm{Kn}$ and therefore spin effects are at least first order in the Knudsen number. In this letter we consider a weakly polarized system by demanding $\chi_{s} \lesssim$ Kn with two expansion parameters $\chi_{s}$ and $\mathrm{Kn}$. For physical observables, we keep zeroth order terms $\mathcal{O}(1)$, first order terms $\mathcal{O}(\mathrm{Kn}), \mathcal{O}\left(\chi_{s}\right)$, as well as second order terms $\mathcal{O}\left(\mathrm{Kn}^{2}\right), \mathcal{O}\left(\mathrm{Kn} \chi_{s}\right)$, and $\mathcal{O}\left(\chi_{s}^{2}\right)$

Wigner function in thermal equilibrium. The distribution function $f(t, \mathbf{x}, \mathbf{p})$ for a classical particle is the probability of finding a particle with the momentum $\mathbf{p}$ at the space-time point $(t, \mathbf{x})$. However, the position and the momentum of a quantum particle can not be determined simultaneously according to the uncertainty principle. Thus the quantum analogue of $f(t, \mathbf{x}, \mathbf{p})$ is the covariant Wigner function defined as a two-point correlation function $[57,58]$

$$
W(x, p) \equiv \int \frac{d^{4} y}{(2 \pi)^{4}} e^{-i p \cdot y}\left\langle: \bar{\psi}\left(x+\frac{y}{2}\right) \otimes \psi\left(x-\frac{y}{2}\right):\right\rangle,
$$

where $\langle::\rangle$ denotes the ensemble average and $\otimes$ denotes the tensor product of two matrices, i.e., $[A \otimes B]_{\alpha \beta}=$ $A_{\beta} B_{\alpha}$. One can verify that $x^{\mu}$ is commutable with $p^{\mu}$ because $x^{\mu}$ is the central position of two points, while $p^{\mu}$ is the momentum conjugate to the relative distance between two points. This indicates that the Wigner function is well-defined in phase space. Note that we neglected gauge fields such as electromagnetic fields throughout this paper. If a gauge field is consid- ered, one should put a gauge link between $\bar{\psi}(x+y / 2)$ and $\psi(x-y / 2)$ as in Ref. [57, 59].

The kinetic equation of the Wigner function can be derived from the Dirac equation [58, 59],

$$
\left[\gamma_{\mu}\left(p^{\mu}+\frac{i}{2} \partial^{\mu}\right)-m\right] W(x, p)=0 .
$$

At zeroth order in gradient, the Wigner function has the following solution,

$$
\begin{aligned}
W_{0}(x, p) & =\frac{1}{(2 \pi)^{3}} \delta\left(p^{2}-m^{2}\right) \\
& \times \sum_{r s}\left\{\theta\left(p^{0}\right)\left[\bar{u}_{s}(\mathbf{p}) \otimes u_{r}(\mathbf{p})\right] f_{r s}^{+}(x, \mathbf{p})\right. \\
& \left.-\theta\left(-p^{0}\right)\left[\bar{v}_{s}(-\mathbf{p}) \otimes v_{r}(-\mathbf{p})\right] f_{r s}^{-}(x,-\mathbf{p})\right\},
\end{aligned}
$$

which can be obtained by using the quantized form of the free Dirac field $\psi(x)$ and $\bar{\psi}(x)$ in Eq. (2). The matrix-valued distributions $f_{r s}^{ \pm}(x, \mathbf{p})$ are constructed by ensemble averages of creation and annihilation operators for particles and antiparticles [60, 61]. The trace $\sum_{s} f_{s s}^{ \pm}(x, \mathbf{p})$ is identified as the number density in phase space for particles/antiparticles. A projection of $f_{r s}^{ \pm}(x, \mathbf{p})$ onto Pauli matrices gives the polarization density in the local rest frame. These distributions satisfy the kinetic equations

$$
\bar{p} \cdot \partial f_{r s}^{ \pm}(x, \mathbf{p})=0,
$$

where $\bar{p}^{\mu} \equiv\left(E_{\mathbf{p}}, \mathbf{p}\right)$ with $E_{\mathbf{p}}=\sqrt{\mathbf{p}^{2}+m^{2}}$. Equations (3) and (5) do not contain collision terms that have been derived in Ref. [62-65]. In this paper, collision terms are neglected because we focus on systems in global or local thermal equilibrium.

The first and second order corrections in space-time gradient for the Wigner function can be obtained by solving the kinetic equation (3),

$$
\begin{aligned}
\delta W(x, p) & =\frac{i}{4 m}\left[\gamma^{\mu}, \partial_{\mu} W_{0}(x, p)\right] \\
& +\frac{1}{16 m^{2}}(\gamma \cdot \partial) W_{0}(x, p)(\gamma \cdot \overleftarrow{\partial}) \\
& +\frac{\gamma \cdot p+m}{8 m\left(p^{2}-m^{2}\right)} \partial^{2} W_{0}(x, p) .
\end{aligned}
$$

So the full solution of Eq. (3) is $W=W_{0}+\delta W$. The expansion in space-time gradient is equivalent to that in the Planck constant $\hbar$. The first order contribution agrees with the results in Refs. [66-69].

The appearance of $\delta W$ is a result of the uncertainty principle for quantum particles. If a system consists of point particles, the Wigner function will be given by $W_{0}$ only, with the momentum being constrained by the normal mass-shell condition $p^{2}=m^{2}$. However, for quantum particles with finite space volume due to the constraint of the uncertainty principle, even though we neglect interactions among particles, the Wigner function contains 
corrections from non-local correlation, described by $\delta W$ in Eq. (6). These corrections include the electric dipole moment induced by an inhomogeneous charge distribution, the magnetization current, and the off-mass-shell correction. The first two corrections are widely known and can be found even in classical electrodynamics [70]. The off-mass-shell correction takes the form of a second order space-time derivative, which can also be obtained from the mass-shell equation of the Wigner function $\left(p^{2}-m^{2}-\partial^{2} / 4\right) W(x, p)=0$. It has been recognized many years ago $[58,59]$ but is rarely studied when the system varies rapidly within the Compton wavelength, i.e., when $\mathrm{Kn} \ll 1$. In our power counting scheme, we identify them as second order corrections $\mathcal{O}\left(\mathrm{Kn}^{2}\right)$.

In thermal equilibrium, we assume $f_{r s}^{ \pm}(x, \mathbf{p})$ have the following form as in Ref. [9],

$$
\begin{aligned}
& f_{\mathrm{eq}, r s}^{+}(x, \mathbf{p})=\frac{1}{2 m} \bar{u}_{r}(\mathbf{p})\left(e^{\beta \cdot \bar{p}-\xi-\frac{1}{4} \omega^{\mu \nu} \sigma_{\mu \nu}}+1\right)^{-1} u_{s}(\mathbf{p}), \\
& f_{\mathrm{eq}, r s}^{-}(x, \mathbf{p})=-\frac{1}{2 m} \bar{v}_{r}(\mathbf{p})\left(e^{\beta \cdot \bar{p}+\xi+\frac{1}{4} \omega^{\mu \nu} \sigma_{\mu \nu}}+1\right)^{-1} v_{s}(\mathbf{p}),
\end{aligned}
$$

where $\bar{p}^{\mu} \equiv\left(E_{\mathbf{p}}, \mathbf{p}\right)$ for both particles and antiparticles, $\sigma^{\mu \nu} \equiv(i / 2)\left[\gamma^{\mu}, \gamma^{\nu}\right]$ with $\gamma^{\mu}$ being gamma matrices. The parameters in Eq. (7) are given by $\beta^{\mu} \equiv \beta u^{\mu}, \xi \equiv \beta \mu$, and $\omega^{\mu \nu} \equiv \beta \bar{\omega}^{\mu \nu}$, with $\beta, u^{\mu}, \mu$, and $\bar{\omega}^{\mu \nu}$ being the inverse temperature, the flow velocity, the chemical potential, and the spin potential, respectively. In global equilibrium, these thermodynamical parameters are space-time constants and then Eq. (7) can be derived rigorously from quantum statistics [9]. We assume that in local equilibrium, the distributions have the same expressions as those in global equilibrium but with space-time depen$\operatorname{dent} \beta^{\mu}(x), \xi(x)$, and $\omega^{\mu \nu}(x)$.

We note that the spin degrees of freedom are dissipative in the quark-gluon plasma [65, 71, 72]. If the relaxation time of spin is much shorter than the typical time scale of the fluid evolution, the considered system will be more close to an instantaneous thermal equilibrium state in Eq. 7. However the system can be off-equilibrium if the relaxation time of spin is relatively large. The relaxation time of spin is recently calculated in strongly coupled regime using the AdS/CFT correspondence [71], or the perturbative QCD [72]. In this study, we assume that the spin relaxation is sufficiently fast, so the system is always in local equilibrium. This is in analogy to the case of ideal hydrodynamics, but equilibrium distributions in Eq. (7) contain spin polarizations described by the spin potential $\bar{\omega}^{\mu \nu}$.

In later calculation, we focus on a weakly polarized system satisfying $\chi_{s} \lesssim K n \ll 1$. Then we can make expansions for thermodynamical parameters:

$$
\begin{aligned}
\omega^{\mu \nu} & =\omega^{(1) \mu \nu}+\omega^{(2) \mu \nu}+\cdots, \\
\beta^{\mu} & =\beta^{(0) \mu}+\beta^{(1) \mu}+\cdots, \\
\xi & =\xi^{(0)}+\xi^{(1)}+\cdots,
\end{aligned}
$$

where ' $(i)^{\prime}$ ' with $i=0,1,2, \cdots$ in superscripts denote orders in $\mathrm{Kn}$ and $\chi_{s}$. Note that the order of magnitude of $\chi_{s}$ is determined by $\omega^{\mu \nu}$ in thermal equilibrium, which will be shown in later calculations, therefore the spin potential is at least first order $\omega^{\mu \nu} \sim \mathcal{O}\left(\chi_{s}\right)$ and its spacetime derivative $\partial^{\alpha} \omega^{\mu \nu} \sim \mathcal{O}\left(\operatorname{Kn} \chi_{s}\right)$ is at least second order. We also expand the distributions in Eq. (7) up to second order in $\chi_{s}$ in Boltzmann limit,

$$
\begin{aligned}
& f_{\mathrm{eq}, r s}^{+}(x, \mathbf{p})=\exp (-\beta \cdot \bar{p}+\xi) \\
& \quad \times\left[\left(1+\frac{1}{16} \omega^{\alpha \beta} \omega_{\alpha \beta}\right) \delta_{r s}+\frac{1}{8 m} \omega^{\mu \nu} \bar{u}_{r}(\mathbf{p}) \sigma_{\mu \nu} u_{s}(\mathbf{p})\right], \\
& f_{\mathrm{eq}, r s}^{-}(x, \mathbf{p})=\exp (-\beta \cdot \bar{p}-\xi) \\
& \quad \times\left[\left(1+\frac{1}{16} \omega^{\alpha \beta} \omega_{\alpha \beta}\right) \delta_{r s}+\frac{1}{8 m} \omega^{\mu \nu} \bar{v}_{r}(\mathbf{p}) \sigma_{\mu \nu} v_{s}(\mathbf{p})\right] .
\end{aligned}
$$

The Wigner function in local equilibrium can be obtained by inserting Eq. (9) into the zeroth order Wigner function (4) and higher order one (6).

Hydrodynamical quantities. According to its definition, the Wigner function satisfies $W^{\dagger}=\gamma^{0} W \gamma^{0}$ and thus can be decomposed in terms of the generators of the Clifford algebra $\Gamma_{i}=\left\{1, i \gamma^{5}, \gamma^{\mu}, \gamma^{5} \gamma^{\mu}, \sigma^{\mu \nu}\right\}$,

$$
W=\frac{1}{4}\left(\mathcal{F}+i \gamma^{5} \mathcal{P}+\gamma^{\mu} \mathcal{V}_{\mu}+\gamma^{5} \gamma^{\mu} \mathcal{A}_{\mu}+\frac{1}{2} \sigma^{\mu \nu} \mathcal{S}_{\mu \nu}\right) \text {. }
$$

where 1 is the $4 \times 4$ unit matrix, $\gamma^{\mu}$ are gamma matrices, $\gamma^{5} \equiv i \gamma^{0} \gamma^{1} \gamma^{2} \gamma^{3}$, and $\sigma^{\mu \nu} \equiv(i / 2)\left[\gamma^{\mu}, \gamma^{\nu}\right]$. The expansion coefficients $\mathcal{F}, \mathcal{P}, \mathcal{V}_{\mu}, \mathcal{A}_{\mu}$, and $\mathcal{S}_{\mu \nu}$ are the scalar, pseudoscalar, vector, axial vector, and tensor components of the Wigner function, respectively, and they are all real [59]. The current density $J^{\mu}(x)$, the energy-momentum tensor (density) $T^{\mu \nu}(x)$, and the spin tensor (density) $S^{\lambda, \mu \nu}$ can be obtained from $\mathcal{V}_{\mu}$ and $\mathcal{A}_{\mu}$ as follows

$$
\begin{aligned}
J^{\mu}(x) & =\int d^{4} p \mathcal{V}^{\mu}(x, p), \\
T^{\mu \nu}(x) & =\int d^{4} p p^{\nu} \mathcal{V}^{\mu}(x, p), \\
S^{\lambda, \mu \nu}(x) & =-\frac{1}{2} \epsilon^{\lambda \mu \nu \rho} \int d^{4} p \mathcal{A}_{\rho}(x, p) .
\end{aligned}
$$

Note that when including spin degrees of freedom, the energy-momentum tensor and the spin-tensor have the pseudo-gauge ambiguity [73-75]. Here we adopt the canonical definition in Eqs. $(12,13)$ for $T^{\mu \nu}$ and $S^{\lambda, \mu \nu}$. Generally the canonical form of $T^{\mu \nu}$ contains an antisymmetric part if the spin polarization is nonzero.

In local equilibrium, the Wigner function can be expressed in terms of the distributions (9). It is straightforward to extract the vector component of the Wigner function to obtain $J^{\mu}(x)$ and $T^{\mu \nu}(x)$ through Eqs. $(11,12)$ 


$$
\begin{aligned}
J_{\mathrm{eq}}^{\mu}(x)= & \left(1+\frac{1}{16} \omega^{\alpha \beta} \omega_{\alpha \beta}\right) K_{1} u^{\mu} \sinh \xi-\frac{1}{16 m^{2}} \partial^{2}\left[\left(2 \beta K_{2}-5 K_{1}\right) u^{\mu} \sinh \xi\right] \\
& +\frac{1}{4 m^{2}} \partial_{\nu}\left\{\left[2 u^{[\mu} \omega_{\alpha}^{\nu]} u^{\alpha}\left(K_{2}+\beta^{-1} K_{1}\right)+\omega^{\mu \nu}\left(K_{2}-\beta^{-1} K_{1}\right)\right] \sinh \xi\right\}, \\
T_{\mathrm{eq}}^{\mu \nu}(x)= & \left(1+\frac{1}{16} \omega^{\alpha \beta} \omega_{\alpha \beta}\right)\left(u^{\mu} u^{\nu} K_{2}-\Delta^{\mu \nu} \beta^{-1} K_{1}\right) \cosh \xi+\frac{1}{4 m^{2}} \partial_{\rho}\left(2 \omega_{\alpha}^{[\mu} I^{\rho] \nu \alpha}+\omega^{\mu \rho} u^{\nu} m^{2} K_{1}\right) \cosh \xi \\
& +\frac{1}{16 m^{2}} \partial^{2}\left\{\left[g^{\mu \nu}\left(2 K_{2}-5 \beta^{-1} K_{1}\right)-u^{\mu} u^{\nu}\left(K_{2}+2 \beta m^{2} K_{1}+\beta^{-1} K_{1}\right)\right] \cosh \xi\right\},
\end{aligned}
$$

where $u^{\mu}$ is the flow velocity and $K_{n}$ are functions of $\beta$ as follows

$$
K_{n}(\beta) \equiv \frac{8}{(2 \pi)^{3}} \int \frac{d^{3} \mathbf{p}}{2 E_{\mathbf{p}}} E_{\mathbf{p}}^{n} e^{-\beta E_{\mathbf{p}}} .
$$

They satisfy the following recursive relation

$$
K_{n}=\frac{n+1}{\beta} K_{n-1}+m^{2} K_{n-2}-\frac{n-2}{\beta} m^{2} K_{n-3},
$$

which allows us to express $K_{n}$ in terms of any $K_{i}$ and $K_{j}$ with $i \neq j$. In Eq. (14) and in the following, we choose $K_{1}$ and $K_{2}$ as basis functions to express $K_{n}$, since $K_{1}$ and $K_{2}$ are related to the particle number and energy density when neglecting second order terms, while other $K_{n}$ do not have explicit physical meanings. The rank-3 moment $I^{\mu \nu \alpha}$ in $T_{\mathrm{eq}}^{\mu \nu}$ in Eq. (14) is given by

$$
\begin{aligned}
I^{\mu \nu \alpha}= & u^{\mu} u^{\nu} u^{\alpha} K_{3}+\frac{1}{3}\left(\Delta^{\mu \nu} u^{\alpha}\right. \\
& \left.+\Delta^{\mu \alpha} u^{\nu}+\Delta^{\nu \alpha} u^{\mu}\right)\left(m^{2} K_{1}-K_{3}\right),
\end{aligned}
$$

where the projection operator is defined as $\Delta^{\mu \nu} \equiv g^{\mu \nu}-$ $u^{\mu} u^{\nu}$.

One can see in Eq. (14) that the spin dependent terms are at least second order in the space-time gradient. At zeroth order in $\mathrm{Kn}$ and $\chi_{s}, J_{\mathrm{eq}}^{\mu}$ and $T_{\mathrm{eq}}^{\mu \nu}$ agree with the results in ideal hydrodynamics [39]. With the help of the fluid velocity $u^{\mu}$, equation (14) can be rewritten as

$$
\begin{aligned}
J_{\mathrm{eq}}^{\mu} & =n_{\mathrm{eq}} u^{\mu}+\delta j^{\mu}, \\
T_{\mathrm{eq}}^{\mu \nu} & =\epsilon_{\mathrm{eq}} u^{\mu} u^{\nu}-P_{\mathrm{eq}} \Delta^{\mu \nu}+\delta T_{S}^{\mu \nu}+\delta T_{A}^{\mu \nu},
\end{aligned}
$$

where $n_{\text {eq }}, \epsilon_{\text {eq }}$, and $P_{\text {eq }}$ are the particle number density, the energy density, and the pressure respectively, and they can be extracted as

$$
\begin{aligned}
n_{\mathrm{eq}} \equiv & u_{\mu} J_{\mathrm{eq}}^{\mu}=\left(1+\frac{1}{16} \omega^{\alpha \beta} \omega_{\alpha \beta}\right) K_{1} \sinh \xi+\frac{1}{4 m^{2}}\left(\nabla_{\mu} u_{\nu}\right) \omega^{\mu \nu}\left(K_{2}+\beta^{-1} K_{1}\right) \sinh \xi \\
& -\frac{1}{2 m^{2}} u_{\mu} \nabla_{\nu}\left[\omega^{\mu \nu} \beta^{-1} K_{1} \sinh \xi\right]-\frac{1}{16 m^{2}} \partial^{2}\left[\left(2 \beta K_{2}-5 K_{1}\right) \sinh \xi\right] \\
\epsilon_{\mathrm{eq}} \equiv & u_{\mu} u_{\nu} T_{\mathrm{eq}}^{\mu \nu}=\left(1+\frac{1}{16} \omega^{\alpha \beta} \omega_{\alpha \beta}\right) K_{2} \cosh \xi+\frac{1}{4 m^{2}}\left(\nabla_{\mu} u_{\nu}\right) \omega^{\mu \nu}\left[5 \beta^{-1}\left(K_{2}+\beta^{-1} K_{1}\right)+m^{2} K_{1}\right] \cosh \xi \\
& -\frac{1}{2 m^{2}} u_{\mu} \nabla_{\nu}\left[\omega^{\mu \nu} \beta^{-1}\left(K_{2}+\beta^{-1} K_{1}\right) \cosh \xi\right]+\frac{1}{16 m^{2}} \partial^{2}\left[\left(K_{2}-6 \beta^{-1} K_{1}-2 \beta m^{2} K_{1}\right) \cosh \xi\right]
\end{aligned}
$$

where $\nabla^{\mu} \equiv \Delta^{\mu \nu} \partial_{\nu}$, and the pressure is obtained by $P_{\text {eq }} \equiv-(1 / 3) \Delta_{\mu \nu} T_{\text {eq }}^{\mu \nu}$, giving the trace anomaly

$$
\begin{aligned}
\epsilon_{\mathrm{eq}}-3 P_{\mathrm{eq}}= & \left(1+\frac{1}{16} \omega^{\alpha \beta} \omega_{\alpha \beta}\right) m^{2} K_{0} \cosh \xi \\
& +\frac{1}{16} \partial^{2}\left[\left(7 K_{0}-2 \beta K_{1}\right) \cosh \xi\right] .
\end{aligned}
$$

The residue terms $\delta j^{\mu}, \delta T_{S}^{\mu \nu}$, and $\delta T_{A}^{\mu \nu}$ in Eq. (18) describe deviations from ideal hydrodynamics without spin.
They are at least second order in the space-time gradient. We identify $\delta j^{\mu}$ as the magnetization current induced by the inhomogeneity of the spin polarization. Here $\delta T_{S}^{\mu \nu}$ and $\delta T_{A}^{\mu \nu}$ are symmetric and antisymmetric respectively for an interchange of $\mu$ and $\nu$. It is straightforward to read out the explicit expressions for $\delta j^{\mu}, \delta T_{S}^{\mu \nu}$, and $\delta T_{A}^{\mu \nu}$ from Eq. (14). They contain the heat flow and viscous tensor correction induced by the spin polarization that have been discussed in the quantum spin vorticity theory 
[76]. In our power counting scheme, these spin-induced terms are of order $\mathcal{O}\left(\mathrm{Kn} \chi_{s}\right)$, which is much smaller than first-order viscous corrections, and comparable to secondorder corrections in regular viscous hydrodynamics without spin. That is because in the quark-gluon plasma produced in heavy-ion collisions, the average spin polarization of particles would be $\sim 0.02$, as indicated by the $\Lambda$ 's global polarization $[5,6]$. The weakly polarized condition $\chi_{s} \lesssim \mathrm{Kn}$ therefore can be fulfilled ( $\mathrm{Kn}$ in heavy-ion collisions has been calculated in Ref. [77]). Note that $\delta j^{\mu}$ and $\delta T_{S}^{\mu \nu}$ also contain second order terms $O\left(\mathrm{Kn}^{2}\right)$. These terms are contributions from long-range correlations, which do not appear in regular ideal hydrodynamics.

Even through we deal with hydrodynamics in local equilibrium, we still obtain viscous terms in Eq. (14). Similar to the viscous hydrodynamics, the definition of the rest frame and $u^{\mu}$ is subtle because the charge and energy currents are in general not parallel when we include higher order corrections. One can either choose the Eckart frame with $u_{E}^{\mu} \equiv J_{\text {eq }}^{\mu} / \sqrt{J_{\text {eq }}^{\nu} J_{\text {eq }, \nu}}$, or the Landau frame with $T_{\mathrm{eq}}^{\mu \nu} u_{L, \nu}=\epsilon_{\mathrm{eq}} u_{L}^{\mu}$. The difference between these two choices is in the second order in Kn and $\chi_{s}$, i.e., $u_{E}^{\mu}-u_{L}^{\mu} \simeq \mathcal{O}\left(\mathrm{Kn}^{2}, \mathrm{Kn} \chi_{s}, \chi_{s}^{2}\right)$.

For the angular momentum, we look at the spin tensor $S^{\lambda, \mu \nu}(x)$ and the dipole-moment tensor $M^{\mu \nu}(x)$. The spin tensor $S^{\lambda, \mu \nu}(x)$ is one part of the total angular momentum tensor $J^{\lambda, \mu \nu}(x)=x^{\mu} T^{\lambda \nu}-x^{\nu} T^{\lambda \mu}+S^{\lambda, \mu \nu}$. Note that the separation of spin and orbital angular momentum is not unique and subject to pseudogauge transformation [73-75]. Here we adopt the canonical definition for $S^{\lambda, \mu \nu}(x)$ as in Eq. (13). In local equilibrium, we can express it in terms of $\beta, \xi, u^{\mu}$, and $\omega^{\mu \nu}$ as

$$
S_{\mathrm{eq}}^{\lambda, \mu \nu}(x)=\frac{1}{4}\left(u^{\lambda} \omega^{\mu \nu}+2 u^{[\mu} \omega^{\nu] \lambda}\right) K_{1} \cosh \xi,
$$

where $K_{1}$ is defined in Eq. (15). The spin potential $\omega^{\mu \nu}$ contains six independent variables. However, $S_{\text {eq }}^{\lambda, \mu \nu}$ only contains three independent variables because it only depends on $\tilde{\omega}^{\mu} \equiv \epsilon^{\mu \nu \alpha \beta} u_{\nu} \omega_{\alpha \beta} / 2$,

$$
S_{\mathrm{eq}}^{\lambda, \mu \nu}(x)=\frac{1}{4} \epsilon^{\lambda \mu \nu \beta} \tilde{\omega}_{\beta} K_{1} \cosh \xi .
$$

In order to study the remaining three degrees of freedom of $\omega^{\mu \nu}$, we look at the dipole momentum tensor defined as

$$
D^{\mu \nu}(x)=\int d^{4} p \mathcal{S}^{\mu \nu}(x, p),
$$

where $\mathcal{S}^{\mu \nu}$ is the tensor component of the Wigner function (10). The dipole moment tensor $D^{\mu \nu}$ is antisymmetric with respect to its indices, which allows the following decomposition

$$
D^{\mu \nu}=\mathcal{E}^{\mu} u^{\nu}-\mathcal{E}^{\nu} u^{\mu}-\epsilon^{\mu \nu \alpha \beta} u_{\alpha} \mathcal{M}_{\beta} .
$$

Such a decomposition depends on the choice of the frame velocity $u^{\mu}$. We identify $\mathcal{E}^{\mu}=D^{\mu \nu} u_{\nu}$ as the electric dipole moment vector and $\mathcal{M}^{\mu}=-(1 / 2) \epsilon^{\mu \nu \alpha \beta} u_{\nu} D_{\alpha \beta}$ as the magnetic dipole moment vector, because they are coupled with electric field and magnetic field respectively if we consider an external electromagnetic field as in Ref. [61]. In local equilibrium, we obtain

$$
\begin{aligned}
\mathcal{E}_{\mathrm{eq}}^{\mu}= & -\frac{1}{m \beta}\left(\omega^{\mu \nu} u_{\nu}+\frac{1}{2} \beta u_{\nu} \partial^{\nu} u^{\mu}\right) K_{1} \sinh \xi \\
& +\frac{1}{2 m} \nabla^{\mu}\left(K_{1} \sinh \xi\right), \\
\mathcal{M}_{\mathrm{eq}}^{\mu}= & -\frac{1}{2 m} \tilde{\omega}^{\mu}\left(K_{2}-\beta^{-1} K_{1}\right) \sinh \xi \\
& -\frac{1}{2 m} \epsilon^{\mu \nu \alpha \beta} u_{\nu}\left(\partial_{\alpha} u_{\beta}\right) K_{1} \sinh \xi .
\end{aligned}
$$

The magnetic dipole moment consists of two parts, one is proportional to $\tilde{\omega}^{\mu}$, which is the contribution of intrinsic spin degrees of freedom; the other part arises from the rotation of the fluid or the orbital angular momentum proportional to the vorticity $\epsilon^{\mu \nu \alpha \beta} u_{\nu}\left(\partial_{\alpha} u_{\beta}\right)$. The electric dipole moment, on the other hand, has three parts: the electric dipole moment of particles, the acceleration of the fluid, and the contribution of the inhomogeneous particle distribution, which are proportional to $\omega^{\mu \nu} u_{\nu}, u_{\nu} \partial^{\nu} u^{\mu}$, and $\nabla^{\mu} n_{\text {eq }}$, respectively. In local equilibrium, a nonvanishing electric dipole moment is allowed.

EOMs for thermodynamical parameters. For the ideal hydrodynamics without spin, the current density $J^{\mu}(x)$ and the energy-momentum tensor $T^{\mu \nu}(x)$ are conserved respectively,

$$
\begin{aligned}
\partial_{\mu} J^{\mu}(x) & =0, \\
\partial_{\nu} T^{\mu \nu}(x) & =0 .
\end{aligned}
$$

These conservation laws still hold in the presence of spin degrees of freedom. Inserting the equilibrium expressions of $J^{\mu}(x)$ and $T^{\mu \nu}(x)$ in Eq. (14) into the conservation laws (26), we derive the equations of motions for $\beta, \xi$, and $u^{\mu}$,

$$
\begin{aligned}
\dot{\beta} & =\frac{K_{2}+\beta^{-1} K_{1} \cosh ^{2} \xi}{K_{1} K_{3} \cosh ^{2} \xi-K_{2} K_{2} \sinh ^{2} \xi} K_{1} \theta \\
\dot{\xi} & =\frac{\left(K_{2}+\beta^{-1} K_{1}\right) K_{2}-K_{1} K_{3}}{K_{1} K_{3} \cosh ^{2} \xi-K_{2} K_{2} \sinh ^{2} \xi} \theta \sinh \xi \cosh \xi \\
\dot{u}^{\mu} & =\frac{K_{1}}{K_{1}+\beta K_{2}} \tanh \xi \nabla^{\mu} \xi-\frac{1}{\beta} \nabla^{\mu} \beta
\end{aligned}
$$

where dot represents the time derivative in comoving frame defined as $d / d \tau \equiv u_{\mu} \partial^{\mu}$, and $\nabla^{\mu} \equiv \Delta^{\mu \nu} \partial_{\nu}$ is the space derivative. With the help of Eqs. (19) and (20), we find that $K_{1} \simeq n_{\mathrm{eq}} / \sinh \xi \simeq \beta P_{\mathrm{eq}} / \cosh \xi$, $K_{2} \simeq \epsilon_{\mathrm{eq}} / \cosh \xi$, with corrections to $K_{1}$ and $K_{2}$ are at least second order in $\mathrm{Kn}$ and $\chi_{s}$, and thus $K_{3}=$ 
$\frac{3}{\beta}\left(K_{2}+\beta^{-1} K_{1}\right)+m^{2} K_{1}$ according to the recurrence relation (16). Therefore, equations in (27) can be expressed in term of the equilibrium quantities $n_{\mathrm{eq}}, P_{\mathrm{eq}}$, and $\epsilon_{\mathrm{eq}}$. Once we do this, we will find that a nonzero $\omega^{\mu \nu}$ does not contribute at the leading and next-to-leading order in Kn and $\chi_{s}$. Such a conclusion can be obtained from another point of view from $J_{\text {eq }}^{\mu}$ and $T_{\text {eq }}^{\mu \nu}$ : the deviation from ideal hydrodynamics without spin is at least $\mathcal{O}\left(\mathrm{Kn}^{2}, \mathrm{Kn} \chi_{s}, \chi_{s}^{2}\right)$.

On the other hand, the conservation of the total angular momentum gives

$$
\partial_{\lambda} S^{\lambda, \mu \nu}(x)=T^{\nu \mu}(x)-T^{\mu \nu}(x) .
$$

Inserting equilibrium expressions in Eqs. $(14,21)$ into the above equation, we obtain the equation of motion for $\omega^{\mu \nu}$ in $\dot{\omega}^{\mu \nu}$, which can be decomposed into

$$
\dot{\omega}^{\mu \nu}=\Delta_{\alpha}^{\mu} \Delta_{\beta}^{\nu} \dot{\omega}^{\alpha \beta}-u^{\mu} \dot{\omega}^{\nu \alpha} u_{\alpha}+u^{\nu} \dot{\omega}^{\mu \alpha} u_{\alpha}
$$

where the first term and $\dot{\omega}^{\mu \nu} u_{\nu}$ appearing in last two terms are given by

$$
\begin{aligned}
\Delta_{\alpha}^{\mu} \Delta_{\beta}^{\nu} \dot{\omega}^{\alpha \beta}= & C_{3} \Delta_{\alpha}^{\mu} \Delta_{\beta}^{\nu} \omega^{\alpha \beta}+C_{4} \Delta_{\beta}^{[\mu} \sigma_{h}^{\nu] \rho} \omega_{\rho}^{\beta} \\
& -\frac{1}{2} C_{4}\left(\nabla^{[\mu} \omega^{\nu]}\right) u^{\rho}+C_{2} C_{4} u^{\rho} \omega^{[\mu}{ }_{\rho}^{\nu]} \xi \\
\dot{\omega}^{\mu \nu} u_{\nu}= & C_{1} \omega^{\mu \nu} u_{\nu}+C_{2} \Delta_{\rho}^{\mu} \omega^{\rho \nu} \nabla_{\nu} \xi \\
& +\sigma_{h}^{\mu \nu} \omega_{\nu \rho} u^{\rho}+\frac{1}{2} \Delta_{\rho}^{\mu}\left(\nabla^{\nu} \omega^{\rho}{ }_{\nu}\right)
\end{aligned}
$$

where $\sigma_{h}^{\mu \nu} \equiv\left(\nabla^{\mu} u^{\nu}+\nabla^{\nu} u^{\mu}\right) / 2-\Delta^{\mu \nu} \theta / 3$. The transport coefficients $C_{i}(i=1,2,3,4)$ are given by

$$
\begin{aligned}
C_{1}= & -\frac{5}{3} \theta+\frac{5}{\beta} \dot{\beta}+\frac{\beta m^{2} K_{1}}{K_{1}+\beta K_{2}} \dot{\beta}-\tanh \xi \dot{\xi}, \\
C_{2}= & \frac{1}{2}\left(1-\frac{5 K_{1}}{K_{1}+\beta K_{2}}-\frac{m^{2} \beta^{2} K_{1} K_{1}}{\left(K_{1}+\beta K_{2}\right)^{2}}\right) \tanh \xi, \\
C_{3}= & -\left[5-\frac{2 \beta^{2} m^{2} K_{1}}{2\left(K_{1}+\beta K_{2}\right)+\beta^{2} m^{2} K_{1}}\right] \frac{\theta}{3} \\
& -\tanh \xi \dot{\xi}+\left[5-\frac{m^{2} \beta^{2}\left(3 K_{1}-\beta K_{2}\right)}{2\left(K_{1}+\beta K_{2}\right)+\beta^{2} m^{2} K_{1}}\right] \frac{\dot{\beta}}{\beta}, \\
C_{4}= & \frac{\beta^{2} m^{2} K_{1}}{2\left(K_{1}+\beta K_{2}\right)+\beta^{2} m^{2} K_{1}}-1 .
\end{aligned}
$$

In our power counting scheme, we have $\omega^{\mu \nu} \sim \mathcal{O}\left(\chi_{s}\right)$ and thus both sides of Eq. (29) are $\mathcal{O}\left(\mathrm{Kn} \chi_{s}\right)$. Equation (29) has a trivial solution $\omega^{\mu \nu}=0$, corresponding to the spinless case. Note that similar results have been derived in Ref. [51], but the transport coefficients are slightly different. That is because we include spin-induced energy current, which leads to an antisymmetric part in $T_{\mathrm{eq}}^{\mu \nu}$, while $T_{\mathrm{eq}}^{\mu \nu}$ used in Ref. [51] is symmetric.
Summary. In this paper, we formulate ideal hydrodynamics with spin degrees of freedom from the Wigner function approach. A general solution to the kinetic equation for the Wigner function has been found up to the second order in space-time gradient. The solution contains a zeroth order part $W_{0}$ and a high-order correction part $\delta W$. The zeroth order part $W_{0}$ can be expressed in terms of matrix-valued distributions, while $\delta W$ can be expressed by space-time derivatives of $W_{0}$. We adopt the equilibrium form of the matrix-valued distributions for particles and antiparticles proposed in Ref. [9].

Based on the Wigner function in local equilibrium, we derive hydrodynamical quantities: the current density, the energy-momentum tensor, the spin tensor, and the electric/magnetic dipole moment. Comparing with ideal hydrodynamics without spin, the current density and the energy-momentum tensor contain additional contributions at the second order in the Knudsen number $\mathrm{Kn}$ and the average spin polarization $\chi_{s}$ from space-time gradients and from spin degrees of freedom respectively. We also find that the spin tensor and magnetic dipole moment depend on $\epsilon^{\mu \nu \alpha \beta} u_{\nu} \omega_{\alpha \beta}$ but does not depend on $\omega^{\mu \nu} u_{\nu}$, while the electric dipole moment is related to $\omega^{\mu \nu} u_{\nu}$. We also derive equations of motions for thermodynamical parameters $\beta, u^{\mu}, \xi$, and $\omega^{\mu \nu}$ from conservation laws. These equations can be applied to numerical simulations for the spin evolution in the quark-gluon plasma. We emphasize that this work is based on the assumption of local equilibrium. In more realistic cases, whether the relaxation time of spin is short enough or not is still under debate. On the other hand, ideal hydrodynamics without spin is acausal and not well-defined, and ideal spin hydrodynamics may also have similar problems [78]. To avoid these problems, dissipative effects induced by particle collisions have to be included.

The authors thank Long-Gang Pang and Shi $\mathrm{Pu}$ for helpful discussions. H.-H. P. and Q.W. are supported in part by the National Natural Science Foundation of China (NSFC) under Grants 11890713 (a sub-grant of 11890710) and 11947301, and by the Strategic Priority Research Program of Chinese Academy of Sciences under Grant XDB34030102. X.-L. S. is supported by the National Natural Science Foundation of China (NSFC) under grants 11935007, 11221504, 11861131009, 11890714 (a sub-grant of 11890710) and 12047528.

[1] Z.-T. Liang and X.-N. Wang, Phys. Rev. Lett. 94, 102301 (2005), [Erratum: Phys.Rev.Lett. 96, 039901 (2006)], nucl-th/0410079.

[2] S. A. Voloshin (2004), nucl-th/0410089.

[3] A. Einstein and W. J. de Hass, Dtsch. Phys. Ges. Verh. p. 152 (1915).

[4] S. J. Barnett, Rev. Mod. Phys. 7, 129 (1935). 
[5] L. Adamczyk et al. (STAR), Nature 548, 62 (2017), 1701.06657.

[6] J. Adam et al. (STAR), Phys. Rev. C98, 014910 (2018), 1805.04400 .

[7] F. Becattini, F. Piccinini, and J. Rizzo, Phys. Rev. C77, 024906 (2008), 0711.1253.

[8] F. Becattini, L. Csernai, and D. J. Wang, Phys. Rev. C88, 034905 (2013), [Erratum: Phys. Rev.C93,no.6,069901(2016)], 1304.4427.

[9] F. Becattini, V. Chandra, L. Del Zanna, and E. Grossi, Annals Phys. 338, 32 (2013), 1303.3431.

[10] I. Karpenko and F. Becattini, Eur. Phys. J. C 77, 213 (2017), 1610.04717.

[11] Y. Xie, D. Wang, and L. P. Csernai, Phys. Rev. C 95, 031901 (2017), 1703.03770.

[12] H. Li, L.-G. Pang, Q. Wang, and X.-L. Xia, Phys. Rev. C 96, 054908 (2017), 1704.01507.

[13] Y. Sun and C. M. Ko, Phys. Rev. C 96, 024906 (2017), 1706.09467.

[14] D.-X. Wei, W.-T. Deng, and X.-G. Huang, Phys. Rev. C 99, 014905 (2019), 1810.00151.

[15] F. Becattini and I. Karpenko, Phys. Rev. Lett. 120, 012302 (2018), 1707.07984.

[16] J. Adam et al. (STAR), Phys. Rev. Lett. 123, 132301 (2019), 1905.11917.

[17] H.-Z. Wu, L.-G. Pang, X.-G. Huang, and Q. Wang, Phys. Rev. Research. 1, 033058 (2019), 1906.09385.

[18] W. Florkowski, A. Kumar, R. Ryblewski, and A. Mazeliauskas, Phys. Rev. C100, 054907 (2019), 1904.00002.

[19] S. Y. F. Liu, Y. Sun, and C. M. Ko (2019), 1910.06774.

[20] S. Y. F. Liu and Y. Yin (2021), 2103.09200.

[21] B. Fu, S. Y. F. Liu, L. Pang, H. Song, and Y. Yin (2021), 2103.10403.

[22] F. Becattini, M. Buzzegoli, and A. Palermo (2021), 2103.10917.

[23] F. Becattini, M. Buzzegoli, A. Palermo, G. Inghirami, and I. Karpenko (2021), 2103.14621.

[24] C. Yi, S. Pu, and D.-L. Yang (2021), 2106.00238.

[25] D. Kharzeev, Phys. Lett. B 633, 260 (2006), hep$\mathrm{ph} / 0406125$.

[26] D. E. Kharzeev, L. D. McLerran, and H. J. Warringa, Nucl. Phys. A803, 227 (2008), 0711.0950.

[27] K. Fukushima, D. E. Kharzeev, and H. J. Warringa, Phys. Rev. D78, 074033 (2008), 0808.3382.

[28] S. A. Voloshin, Phys. Rev. Lett. 105, 172301 (2010), 1006.1020.

[29] F.-Q. Wang and J. Zhao, Nucl. Sci. Tech. 29, 179 (2018).

[30] J. Adam et al. (STAR), Nucl. Sci. Tech. 32, 48 (2021), 1911.00596.

[31] M. Abdallah et al. (STAR) (2021), 2109.00131.

[32] Y.-C. Liu and X.-G. Huang, Nucl. Sci. Tech. 31, 56 (2020), 2003.12482.

[33] J.-H. Gao, G.-L. Ma, S. Pu, and Q. Wang, Nucl. Sci. Tech. 31, 90 (2020), 2005.10432.

[34] J.-H. Gao, Z.-T. Liang, Q. Wang, and X.-N. Wang (2020), 2009.04803.

[35] J.-H. Gao, Z.-T. Liang, and Q. Wang, Int. J. Mod. Phys. A 36, 2130001 (2021), 2011.02629.

[36] P. Romatschke, Int. J. Mod. Phys. E 19, 1 (2010), 0902.3663.

[37] U. Heinz and R. Snellings, Ann. Rev. Nucl. Part. Sci. 63, 123 (2013), 1301.2826.

[38] C. Gale, S. Jeon, and B. Schenke, Int. J. Mod. Phys. A 28, 1340011 (2013), 1301.5893.
[39] P. Romatschke and U. Romatschke, Relativistic Fluid Dynamics In and Out of Equilibrium, Cambridge Monographs on Mathematical Physics (Cambridge University Press, 2019), ISBN 978-1-108-48368-1, 978-1-108-75002-8, 1712.05815.

[40] C. Shen and L. Yan, Nucl. Sci. Tech. 31, 122 (2020), 2010.12377.

[41] S. Wu, C. Shen, and H. Song (2021), 2104.13250.

[42] J. Weyssenhoff and A. Raabe, Acta Phys. Polon. 9, 7 (1947).

[43] F. Becattini and L. Tinti, Annals Phys. 325, 1566 (2010), 0911.0864.

[44] D. Montenegro, L. Tinti, and G. Torrieri, Phys. Rev. D 96, 056012 (2017), [Addendum: Phys.Rev.D 96, 079901 (2017)], 1701.08263.

[45] D. Montenegro, L. Tinti, and G. Torrieri, Phys. Rev. D 96, 076016 (2017), 1703.03079.

[46] W. Florkowski, B. Friman, A. Jaiswal, and E. Speranza, Phys. Rev. C97, 041901 (2018), 1705.00587.

[47] W. Florkowski, B. Friman, A. Jaiswal, R. Ryblewski, and E. Speranza, Phys. Rev. D97, 116017 (2018), 1712.07676.

[48] W. Florkowski, A. Kumar, and R. Ryblewski, Prog. Part. Nucl. Phys. 108, 103709 (2019), 1811.04409.

[49] W. Florkowski, Acta Phys. Polon. B 50, 1047 (2019), 1904.01840.

[50] A. D. Gallegos, U. Gürsoy, and A. Yarom (2021), 2101.04759.

[51] S. Bhadury, W. Florkowski, A. Jaiswal, A. Kumar, and R. Ryblewski, Phys. Rev. D 103, 014030 (2021), 2008.10976.

[52] S. Bhadury, W. Florkowski, A. Jaiswal, A. Kumar, and R. Ryblewski, Phys. Lett. B 814, 136096 (2021), 2002.03937.

[53] K. Hattori, M. Hongo, X.-G. Huang, M. Matsuo, and H. Taya, Phys. Lett. B 795, 100 (2019), 1901.06615.

[54] K. Fukushima and S. Pu, Phys. Lett. B 817, 136346 (2021), 2010.01608.

[55] S. Li, M. A. Stephanov, and H.-U. Yee (2020), 2011.12318.

[56] D. She, A. Huang, D. Hou, and J. Liao (2021), 2105.04060 .

[57] U. W. Heinz, Phys. Rev. Lett. 51, 351 (1983).

[58] H. T. Elze, M. Gyulassy, and D. Vasak, Nucl. Phys. B276, 706 (1986).

[59] D. Vasak, M. Gyulassy, and H. T. Elze, Annals Phys. 173, 462 (1987).

[60] X.-L. Sheng, Ph.D. thesis, Frankfurt U. (2019), 1912.01169.

[61] X.-L. Sheng, Q. Wang, and X.-G. Huang, Phys. Rev. D 102, 025019 (2020), 2005.00204.

[62] D.-L. Yang, K. Hattori, and Y. Hidaka (2020), 2002.02612.

[63] N. Weickgenannt, E. Speranza, X.-l. Sheng, Q. Wang, and D. H. Rischke (2020), 2005.01506.

[64] X.-L. Sheng, N. Weickgenannt, E. Speranza, D. H. Rischke, and Q. Wang (2021), 2103.10636.

[65] Z. Wang and P. Zhuang (2021), 2105.00915.

[66] J.-H. Gao and Z.-T. Liang, Phys. Rev. D100, 056021 (2019), 1902.06510.

[67] N. Weickgenannt, X.-L. Sheng, E. Speranza, Q. Wang, and D. H. Rischke, Phys. Rev. D100, 056018 (2019), 1902.06513.

[68] K. Hattori, Y. Hidaka, and D.-L. Yang, Phys. Rev. D 
100, 096011 (2019), 1903.01653.

[69] Z. Wang, X. Guo, S. Shi, and P. Zhuang, Phys. Rev. D 100, 014015 (2019), 1903.03461.

[70] J. D. Jackson, Classical Electrodynamics (Wiley, 1998), ISBN 978-0-471-30932-1.

[71] S. Li and H.-U. Yee, Phys. Rev. D 98, 056018 (2018), 1805.04057.

[72] S. Li and H.-U. Yee, Phys. Rev. D 100, 056022 (2019), 1905.10463.

[73] F. Becattini and L. Tinti, Phys. Rev. D 87, 025029
(2013), 1209.6212.

[74] K. Fukushima and S. Pu (2020), 2001.00359.

[75] E. Speranza and N. Weickgenannt, Eur. Phys. J. A 57, 155 (2021), 2007.00138.

[76] M. Fukuda, K. Ichikawa, M. Senami, and A. Tachibana, AIP Advances 6, 025108 (2016), ISSN 2158-3226, URL https://dx.doi.org/10.1063/1.4942087.

[77] H. Niemi and G. S. Denicol (2014), 1404.7327.

[78] E. Speranza, F. S. Bemfica, M. M. Disconzi, and J. Noronha (2021), 2104.02110. 